\title{
Panorama de la investigación en redes sociales
}

\author{
José Luis Molina - Departament d'Antropologia social - UAB ${ }^{1}$
}

\section{Resumen}

A través de un análisis de los resultados obtenidos en la Web of Science con la expresión "social network*", la revista Social Networks y la Revista REDES, se proporciona una visión de conjunto de la investigación en este campo. Además, basándose en las investigaciones en marcha sobre redes personales se argumenta la característica dual de las redes sociales, característica que permite un nuevo tratamiento de la dualidad micro-macro y de la cualitativa-cuantitativa.

Palabras clave: redes sociales - análisis de redes sociales - redes personales, bibliometría.

\begin{abstract}
After the query "social network*" in the Web of Science, the articles published in the Journals Social Networks and REDES this paper provides a general overview of this field of research. Moreover, with data about personal networks from the research projects in progress, it is argued that networks exhibit a dual nature that allows a new perspective about the dualities micro-macro and qualitativequantitative.
\end{abstract}

Key words: social networks - social network analysis - personal networks bibliometrics.

Mis primeras palabras son para agradecer la renovada invitación para participar en un seminario del IIMAS de la UNAM. En el 2002 tuve la oportunidad de presentar aquí mismo el primer número de la Revista REDES (http://revista-redes.rediris.es), una publicación orientada al público iberoamericano interesado en la perspectiva de redes. Desde entonces, muchas cosas han cambiado y todas para bien: sólo mencionar que ya disponemos de 12 números publicados con 85 artículos y reseñas, que Isidro Maya Jariego tomó con acierto el relevo en la dirección de la Revista y que ésta empieza a ser indexada en diferentes bases de datos bibliográficas. Hoy, 5 años después, me propongo presentar el conjunto de la investigación existente hoy día en redes y redes sociales. Aunque se trata de un propósito ambicioso, creo que simplemente explotando la información disponible a nuestro alrededor podemos obtener una razonable visión de conjunto. Así, echaremos un vistazo a las publicaciones disponibles en la Web of Science que incluyen la expresión "social network*" en su descripción, al conjunto de publicaciones de la revista Social Networks y, cómo no, al conjunto de lo publicado

\footnotetext{
${ }^{1}$ Enviar correspondencia a: joseluis.molina@uab.es
} 
REDES- Revista hispana para el análisis de redes sociales

Vol.17,\#11, Diciembre 2009

http://revista-redes.rediris.es

en la Revista REDES. Por supuesto, otras fuentes influyentes como manuales y libros pueden contribuir a completar este panorama, pero no hemos tenido oportunidad de realizar una investigación sistemática en este apartado. Por último, basándonos en las investigaciones en marcha en las que tengo el privilegio de participar, realizaré una reflexión sobre el camino a seguir, al menos en una pequeña esquina de este inmenso campo que denominamos genéricamente "redes".

Y empezaremos por ahí. Después de darle vueltas uno se da cuenta que el nexo de unión de toda la producción científica que incorpora la perspectiva de redes es simplemente la palabra "red" (o "redes"). Así, nos encontramos con enfoques que utilizan la palabra red como metáfora de la complejidad, enfoques que desarrollan algoritmos para identificar estructuras en los datos reticulares, enfoques que utilizan las redes como un elemento más del conjunto de estrategias para explicar sus problemas de investigación... Las expresiones "ciencia de las redes", "análisis de redes sociales", "teoría y análisis de redes sociales" reflejan esta pluralidad de enfoques y objetos de investigación. No hay nada malo en ello. Las redes no sólo son buenas para pensar, sino que también son buenas para investigar la realidad (en cualquiera de sus dimensiones) y especialmente buenas para analizar. La complejidad no es un problema. Es su medio natural. Debo a un profesor de informática de la Universidad de Granada, J.J. Merelo esta idea: las redes representan la complejidad. La representación visual no es opcional sino que es constitutiva de la aproximación. Efectivamente, la representación visual tiene la característica de comunicar de forma instantánea un conjunto de variables visuales: la forma, el tamaño, el color, la posición, las relaciones, las etiquetas. Aún a riesgo de repetirme no puedo dejar de recordar que detrás de cada mapa del tiempo que vemos cada día se hallan los modelos matemáticos más complejos ideados por la humanidad y más información de la que podremos recoger en toda nuestra vida. $Y$ al final tienes a una persona sonriendo y moviendo las manos sobre una visualización...

Visualización, algoritmos, humanidades, ciencias sociales, física, biología... todo ello se da cita en el campo de las redes. Un viejo esquema del desarrollo intelectual de la perspectiva de redes puede ser bueno traer aquí otra vez a colación (Ilustración $1)$. 


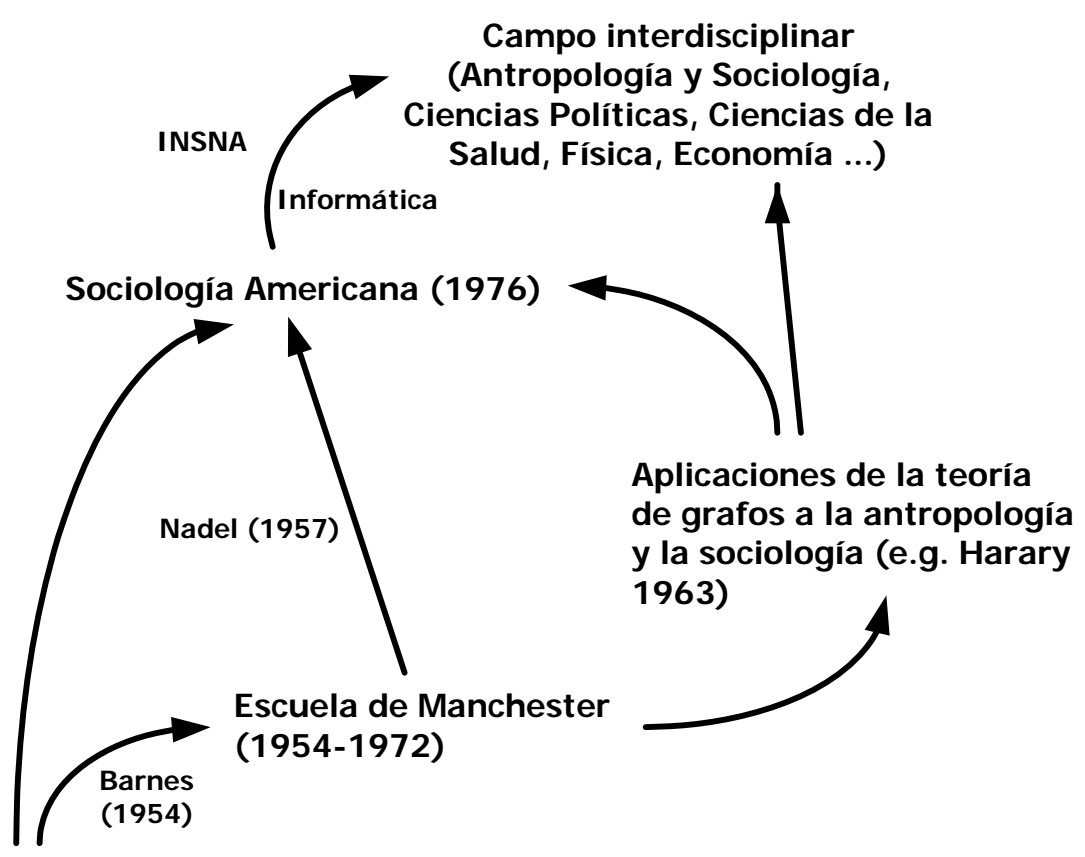

Sociometría de Moreno (1934)

I lustración 1. El desarrollo de las redes sociales (Molina, 2001)

El hecho de combinar matemáticos como Frank Harary (por cierto, no es casualidad que el servidor en el que está alojada la página web que anuncia este coloquio lleve el nombre del insigne académico), con psicólogos, antropólogos y sociólogos y más adelante, con físicos e informáticos, ha dado lugar a este campo tan genuinamente multidisciplinar. Es un valor que no podemos perder. Dicho en términos de redes: el alto grado de intermediación de la disciplina en el panorama de las ciencias y las humanidades nos hace tener ideas nuevas y acceder a recursos y capacidades diferentes. Al mismo tiempo, su carácter interdiciplinar y emergente ha propiciado la colaboración espontánea y omnipresente, propia de los poco institucionalizados o de los que tienen pocos recursos. Esperamos que esta característica, propia también de la filosofía del código abierto u open source, se mantenga en el futuro, aunque no va a ser fácil, la verdad.

Pero entremos en materia. Para ello analizaremos en primer lugar el conjunto de las publicaciones que incorporan la perspectiva de redes en revistas indexadas en el Web of Science.

\section{Las redes en la Web of Science}

Después de introducir la expresión "social network*" en la Web of Science obtuvimos 6.546 referencias. Distribuidas por años obtenemos el siguiente gráfico (Ilustración 2). 
REDES- Revista hispana para el análisis de redes sociales

Vol.17,\#11, Diciembre 2009

http: // revista-redes.rediris.es

Número de artículos referenciados en laWeb of Science de "s ocial network" (1953-2006)

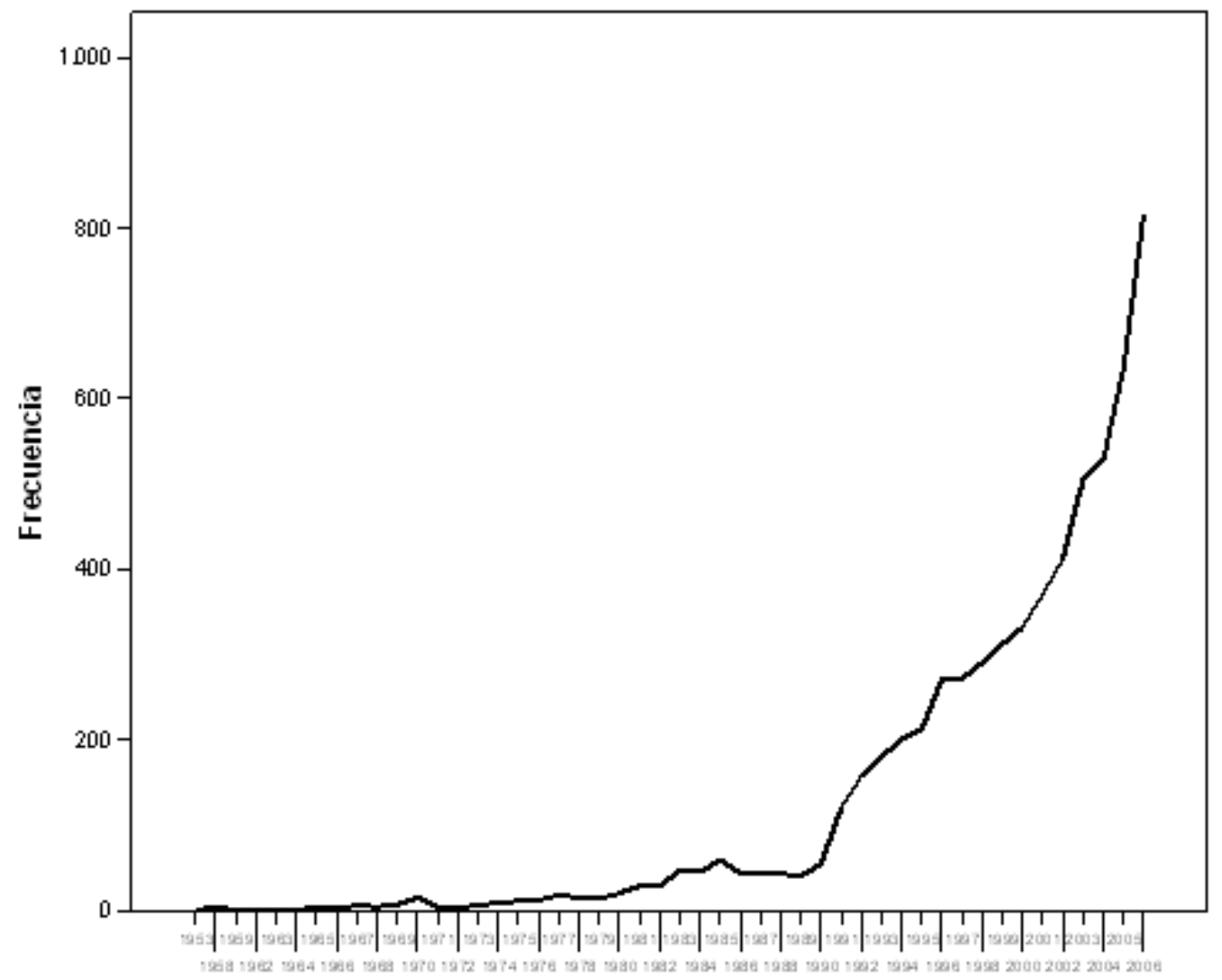

I lustración 2. Distribución de los artículos referenciados en la Web of Science ("social network*", 19532006)

La verdad que la progresión es bastante impresionante, aún teniendo en cuenta que parte del crecimiento se debe a la propia dinámica de la producción científica y su indexación. La misma información pero teniendo en cuenta tanto el número de artículos publicados como el número de citas de los últimos 20 años los tenemos en la llustración 3. 


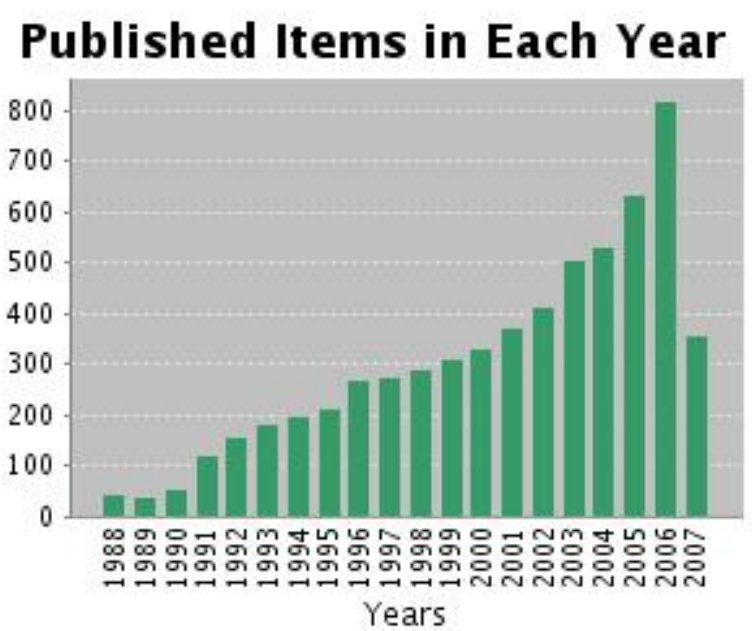

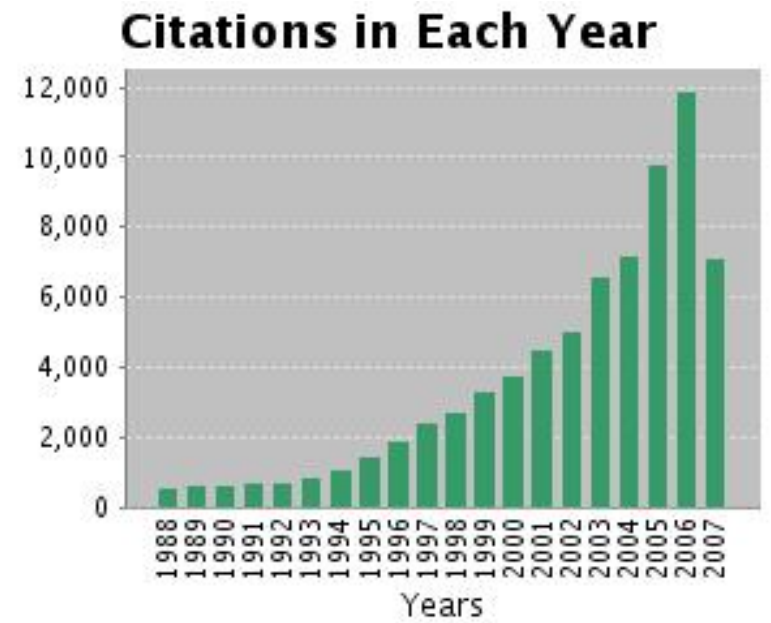

$\mathrm{TS}=($ "social network*") DocType=All document types; Language=All languages; Databases=SClEXPANDED, SSCI, A\&HCI; Timespan=1900-2007

I lustración 3. Publicaciones y citas de los últimos 20 años sobre publicaciones con el descriptor ("social network*).

Evidentemente, la bajada del 2007 se debe al momento en el que se ha realizado la consulta. Obsérvese el salto de los dos últimos años... Ahora bien. ¿Cuáles son los artículos más citados? La respuesta la tenemos en la Tabla 1. 
REDES- Revista hispana para el análisis de redes sociales

Vol.17,\#11, Diciembre 2009

http: //revista-redes.rediris.es

\begin{tabular}{|c|c|c|c|c|c|c|c|}
\hline 6,564 results found & 2003 & 2004 & 2005 & 2006 & 2007 & Total & $\begin{array}{c}\text { Average } \\
\text { Citation } \\
\mathbf{s} \\
\text { per Year }\end{array}$ \\
\hline & 6575 & 7232 & 9830 & 11865 & 7150 & 76,419 & 1389.44 \\
\hline
\end{tabular}

Watts DJ, Strogatz SH

Collective dynamics of 'small-world'

1. networks

NATURE 393 (6684): 440-442 JUN 41998

$\begin{array}{llllll}222 & 339 & 424 & 532 & 252 & \underline{2121}\end{array}$

212.10

BERKMAN LF, SYME SL

SOCI AL NETWORKS, HOST-RESISTANCE,

2. AND MORTALITY - 9-YEAR FOLLOW-UP-

STUDY OF ALAMEDA COUNTY RESIDENTS

AMERICAN J OURNAL OF EPIDEMIOLOGY

109 (2): 186-204 1979

Newman MEJ

The structure and function of complex

3. networks

SIAM REVIEW 45 (2): 167-256 JUN 2003

Albert $\mathrm{R}$, Jeong $\mathrm{H}$, Barabasi $\mathrm{AL}$

Error and attack tolerance of complex

4. networks

NATURE 406 (6794): 378-382 JUL 272000

FREEMAN LC

CENTRALITY IN SOCI AL NETWORKS

5. CONCEPTUAL CLARIFICATION

SOCIAL NETWORKS 1 (3): 215-239 1979

Pastor-Satorras R, Vespignani A

Epidemic spreading in scale-free networks

6. PHYSICAL REVIEW LETTERS 86 (14): 3200-

3203 APR 22001

FURMAN W, BUHRMESTER D

CHILDRENS PERCEPTIONS OF THE

7. PERSONAL RELATIONSHIPS IN THEIR

SOCI AL NETWORKS

DEVELOPMENTAL PSYCHOLOGY 21 (6):

1016-1024 1985

TOLSDORF CC

SOCI AL NETWORKS, SUPPORT, AND

8. COPING - EXPLORATORY-STUDY

FAMILY PROCESS 15 (4): 407-417 1976

\section{2}

$71 \quad 38 \quad \underline{1703}$

58.72

$\begin{array}{lllll}116 & 222 & 315 & 205 & 868\end{array}$

173.60

Taylor SE, Klein LC, Lewis BP, et al.

Biobehavioral responses to stress in

9. females: Tend-and-befriend, not fight-or-

PSYCHOLOGICAL REVIEW 107 (3): 411-

429 JUL 2000

CAIRNS RB, CAIRNS BD, NECKERMAN HJ, et al.

10 SOCI AL NETWORKS AND AGGRESSIVEBEHAVIOR - PEER SUPPORT OR PEER

REJECTION

DEVELOPMENTAL PSYCHOLOGY 24 (6):

815-823 NOV 1988

$90 \quad 117 \quad 139$

148

83

$\underline{713}$

89.12

35

$30 \quad 36$

55

47

$\underline{504}$

17.38

48

$62 \quad 77$

118

56

411

58.71

22

$21 \quad 15$

35

7

$\underline{339}$

14.74

Tabla 1. Los 10 artículos más citados en la Web of Science sobre publicaciones con el descriptor ("social network*). 
REDES- Revista hispana para el análisis de redes sociales

Vol.17,\#11, Diciembre 2009

http: // revista-redes.rediris.es

En la lista nos encontramos con Watts y Strogatz, sociólogos matemáticos, la revista Nature y la feliz operacionalización del concepto de Mundo Pequeño de Milgram (1967). A continuación, encontramos a una mujer, Berkman, de extraordinaria influencia en el mundo de la salud al apoyar empíricamente una y otra vez la relación positiva entre red social y salud. El tercero y el cuartos, Newman y los también físicos Albert, Jeong y Barabasi y sus trabajos sobre redes complejas. En quinto lugar, Freeman, un autor más cercano para algunos de nosotros y su artículo fundacional en Social Networks sobre centralidad. A continuación otros físicos y una serie de psicólogos del comportamiento entre los que cabe destacar el concepto de apoyo social (social support).

Esto es lo que hay. Es verdad que en la Web of Science se hayan infrarepresentadas las contribuciones de las Humanidades y las Ciencias sociales por haberse incorporado más tarde a la dinámica de publicación científica dominante hoy día, basada en artículos de varios autores en revistas indexadas. Pero de entrada vemos que las redes complejas estudiadas básicamente por físicos y la relación entre salud y redes sociales (tanto en la dimensión médica como psicológica) son plenamente dominantes en el conjunto de las publicaciones indexadas en la fuente mencionada.

Esta impresión cuantitativa la tenemos también después de hacer un análisis cualitativo de todos los abstracts con la ayuda del programa de distribución gratuita TextSTAT ${ }^{2}$.

Los resultados, después de eliminar preposiciones, adverbios, formas verbales y palabras relacionadas con el proceso investigador (como "variables", "marco teórico", “discusión", etc.) se pueden encontrar en la Tabla 2.

${ }^{2}$ http://www.niederlandistik.fu-berlin.de/textstat/software-en.html 
REDES- Revista hispana para el análisis de redes sociales

Vol.17,\#11, Diciembre 2009

http://revista-redes.rediris.es

\begin{tabular}{|c|c|c|c|}
\hline Word & $\mathbf{N}$ & Word & $\mathbf{N}$ \\
\hline soci- & 5.113 & education- & 876 \\
\hline support & 4.176 & depressi- & 867 \\
\hline health- & 3.640 & mental & 849 \\
\hline relation- & 3.567 & parent- & 828 \\
\hline famil- & 2.568 & econom- & 824 \\
\hline women & 2.491 & drug & 819 \\
\hline communit- & 2.414 & manage- & 794 \\
\hline individual- & 1.965 & predict- & 793 \\
\hline structur- & 1.910 & physical- & 788 \\
\hline patient & 1.739 & association- & 778 \\
\hline network- & 1.677 & knowledge & 773 \\
\hline risk- & 1.647 & survey & 769 \\
\hline person- & 1.643 & environment- & 746 \\
\hline child- & 1.549 & partner & 739 \\
\hline friend- & 1.542 & peer- & 737 \\
\hline information & 1.468 & practic- & 732 \\
\hline age- & 1.440 & adolescen- & 710 \\
\hline groups & 1.402 & rights & 667 \\
\hline influence- & 1.376 & Elsevier & 661 \\
\hline life & 1.344 & symptoms & 659 \\
\hline theor- & 1.333 & user- & 659 \\
\hline role- & 1.249 & disease & 611 \\
\hline scienc- & 1.247 & gender & 608 \\
\hline behavior- & 1.208 & communication & 607 \\
\hline sex- & 1.191 & cultur- & 601 \\
\hline men & 1.131 & employ- & 601 \\
\hline organization- & 1.103 & ill- & 600 \\
\hline members & 1.097 & dynamic & 596 \\
\hline status & 1.044 & local & 596 \\
\hline system- & 1.036 & size & 591 \\
\hline care & 1.031 & urban & 587 \\
\hline work & 1.020 & link- & 583 \\
\hline capital & 1.012 & psychiatr- & 578 \\
\hline population & 1.003 & school & 546 \\
\hline adult- & 966 & psychological & 540 \\
\hline ties & 962 & human & 539 \\
\hline HIV & 956 & job- & 539 \\
\hline intervention- & 947 & ethn- & 533 \\
\hline treatment & 947 & interaction- & 532 \\
\hline older & 929 & psychosocial & 524 \\
\hline American & 923 & emotional & 518 \\
\hline strategic- & 909 & perception- & 504 \\
\hline
\end{tabular}

Tabla 2. Frecuencia $(>500)$ de sustantivos (eliminados adverbios, preposiciones, formas verbales y palabras relacionadas con la investigación y la publicación científica en general) en los abstracts de los artículos indexados en la Web of Science con el descriptor ("social network*). 
REDES- Revista hispana para el análisis de redes sociales

Vol.17,\#11, Diciembre 2009

http://revista-redes.rediris.es

Si observamos las 10 palabras con más frecuencia de aparición podemos ver cómo al lado de palabras propias de las redes como soci- (social, society, etc.), relationy structur-, aparecen otras que nos remiten al campo de la salud, las redes sociales y el apoyo social: support, health-, famil-, women, communit-, patient.

Por lo que se refiere a las revistas científicas de las que está extraída la información ( 11.800 en total!), podemos encontrar rótulos clasificables en Ciencias Sociales, Salud (en todas sus vertientes), Management, trabajo social ...

\begin{tabular}{|l|c|c|}
\hline Subject Category & N & $\%$ \\
\hline SOCI OLOGY & 936 & 10,4 \\
\hline PUBLIC, ENVI RONMENTAL \& OCCUPATI ONAL HEALTH & 741 & 8,2 \\
\hline PSYCHI ATRY & 544 & 6,0 \\
\hline ANTHROPOLOGY & 376 & 4,2 \\
\hline GERONTOLOGY & 344 & 3,8 \\
\hline PSYCHOLOGY, MULTIDISCI PLINARY & 339 & 3,8 \\
\hline MANAGEMENT & 301 & 3,3 \\
\hline PSYCHOLOGY, DEVELOPMENTAL & 268 & 3,0 \\
\hline PSYCHOLOGY, SOCIAL & 263 & 2,9 \\
\hline PSYCHOLOGY, CLINICAL & 237 & 2,6 \\
\hline SOCIAL SCIENCES, BI OMEDICAL & 227 & 2,5 \\
\hline SOCIAL WORK & 227 & 2,5 \\
\hline SOCIAL SCIENCES, INTERDISCI PLINARY & 213 & 2,4 \\
\hline GERI ATRICS \& GERONTOLOGY & 202 & 2,2 \\
\hline BUSINESS & 201 & 2,2 \\
\hline PSYCHOLOGY & 190 & 2,1 \\
\hline FAMILY STUDIES & 184 & 2,0 \\
\hline SUBSTANCE ABUSE & 181 & 2,0 \\
\hline ECONOMICS & 172 & 1,9 \\
\hline GEOGRAPHY & 149 & 1,6 \\
\hline NURSING & 128 & 1,4 \\
\hline MEDICINE, GENERAL \& INTERNAL & 125 & 1,4 \\
\hline COMPUTER SCI ENCE, THEORY \& METHODS & 120 & 1,3 \\
\hline PLANNI NG \& DEVELOPMENT & 118 & 1,3 \\
\hline REHABILITATION & 118 & 1,3 \\
\hline HEALTH POLICY \& SERVICES & 117 & 1,3 \\
\hline INFECTIOUS DISEASES & 116 & 1,3 \\
\hline COMMUNICATION & 114 & 1,3 \\
\hline ENVI RONMENTAL STUDIES & 114 & 1,3 \\
\hline
\end{tabular}

Tabla 3. Clasificación temática de las revistas científicas que publican el $80 \%$ de artículos con el descriptor ("social network*). 
REDES- Revista hispana para el análisis de redes sociales

Vol.17,\#11, Diciembre 2009

http: //revista-redes.rediris.es

Los autores con más de 10 publicaciones se muestran en la Tabla 4, en la 5 los países y en la 6 las instituciones.

\begin{tabular}{|l|c|c|}
\hline Author & N & $\%$ \\
\hline BERKMAN, LF & 28 & 0,43 \\
\hline LITWI N, H & 24 & 0,37 \\
\hline NEWMAN, MEJ & 24 & 0,37 \\
\hline LATKIN, CA & 23 & 0,35 \\
\hline DOREI AN, P & 21 & 0,32 \\
\hline HANSON, BS & 19 & 0,29 \\
\hline POTTERAT, JJ & 19 & 0,29 \\
\hline FRIEDMAN, SR & 18 & 0,27 \\
\hline PATTISON, P & 18 & 0,27 \\
\hline WELLMAN, B & 18 & 0,27 \\
\hline FARMER, TW & 17 & 0,26 \\
\hline OSTERGREN, PO & 17 & 0,26 \\
\hline WASSERMAN, S & 17 & 0,26 \\
\hline MUTH, SQ & 16 & 0,24 \\
\hline ROTHENBERG, RB & 16 & 0,24 \\
\hline CAIRNS, BD & 15 & 0,23 \\
\hline COHEN, CI & 15 & 0,23 \\
\hline KAWACHI, I & 15 & 0,23 \\
\hline SNIJDERS, TAB & 15 & 0,23 \\
\hline VALENTE, TW & 15 & 0,23 \\
\hline ANTONUCCI, TC & 14 & 0,21 \\
\hline BORGATTI, SP & 14 & 0,21 \\
\hline HANSSON, L & 14 & 0,21 \\
\hline KILDUFF, M & 14 & 0,21 \\
\hline NEAIGUS, A & 14 & 0,21 \\
\hline VLAHOV, D & 14 & 0,21 \\
\hline
\end{tabular}

\begin{tabular}{|l|c|c|}
\hline Author & N & $\%$ \\
\hline BERNARD, HR & 13 & 0,20 \\
\hline COHEN, S & 12 & 0,18 \\
\hline CROSS, R & 12 & 0,18 \\
\hline KILLWORTH, PD & 12 & 0,18 \\
\hline ROBI NS, G & 12 & 0,18 \\
\hline ROTHENBERG, R & 12 & 0,18 \\
\hline SEEMAN, TE & 12 & 0,18 \\
\hline THORNICROFT, G & 12 & 0,18 \\
\hline WATTS, DJ & 12 & 0,18 \\
\hline BOWLING, A & 11 & 0,17 \\
\hline BRASS, DJ & 11 & 0,17 \\
\hline BREWER, DD & 11 & 0,17 \\
\hline FERLIGOJ, A & 11 & 0,17 \\
\hline KELLY, JA & 11 & 0,17 \\
\hline KLOVDAHL, AS & 11 & 0,17 \\
\hline LATKIN, C & 11 & 0,17 \\
\hline MANDELL, W & 11 & 0,17 \\
\hline MCCARTY, C & 11 & 0,17 \\
\hline SUNDQUIST, J & 11 & 0,17 \\
\hline WEISNER, C & 11 & 0,17 \\
\hline CAIRNS, RB & 10 & 0,15 \\
\hline CARLEY, KM & 10 & 0,15 \\
\hline FAUST, K & 10 & 0,15 \\
\hline
\end{tabular}

Tabla 4. Autores con 10 artículos o más indexados en la Web of Science con el descriptor ("social network*). 
REDES- Revista hispana para el análisis de redes sociales

Vol.17,\#11, Diciembre 2009

http: // revista-redes.rediris.es

\begin{tabular}{|l|c|c|}
\hline Country/ Territory & N & \% \\
\hline USA & 3539 & 53,9 \\
\hline ENGLAND & 621 & 9,5 \\
\hline CANADA & 365 & 5,6 \\
\hline GERMANY & 315 & 4,8 \\
\hline SWEDEN & 310 & 4,7 \\
\hline NETHERLANDS & 273 & 4,2 \\
\hline AUSTRALIA & 200 & 3,0 \\
\hline FRANCE & 125 & 1,9 \\
\hline ISRAEL & 109 & 1,7 \\
\hline SPAIN & 104 & 1,6 \\
\hline PEOPLES R CHINA & 100 & 1,5 \\
\hline ITALY & 92 & 1,4 \\
\hline SWITZERLAND & 92 & 1,4 \\
\hline FINLAND & 82 & 1,2 \\
\hline NORWAY & 82 & 1,2 \\
\hline DENMARK & 77 & 1,2 \\
\hline JAPAN & 77 & 1,2 \\
\hline SCOTLAND & 68 & 1,0 \\
\hline BELGIUM & 46 & 0,7 \\
\hline TAIWAN & 44 & 0,7 \\
\hline NEW ZEALAND & 39 & 0,6 \\
\hline AUSTRIA & 37 & 0,6 \\
\hline BRAZIL & 36 & 0,5 \\
\hline MEXICO & 36 & 0,5 \\
\hline IRELAND & 33 & 0,5 \\
\hline SOUTH KOREA & 33 & 0,5 \\
\hline WALES & 29 & 0,4 \\
\hline SINGAPORE & 26 & 0,4 \\
\hline
\end{tabular}

Tabla 5. Los 28 países con más artículos o más indexados en la Web of Science con el descriptor ("social network*).

\begin{tabular}{|l|c|c|}
\hline \multicolumn{1}{|c|}{ I nstitution Name } & N & $\%$ \\
\hline UNIV N CAROLINA & 156 & 2,4 \\
\hline UNIV MICHI GAN & 132 & 2,0 \\
\hline HARVARD UNIV & 119 & 1,8 \\
\hline UNIV I LLI NOIS & 117 & 1,8 \\
\hline UNIV CALIF LOS ANGELES & 109 & 1,7 \\
\hline COLUMBIA UNIV & 97 & 1,5 \\
\hline UNIV WASHINGTON & 97 & 1,5 \\
\hline JOHNS HOPKINS UNIV & 82 & 1,2 \\
\hline UNIV TEXAS & 75 & 1,1 \\
\hline CORNELL UNIV & 74 & 1,1 \\
\hline PENN STATE UNIV & 71 & 1,1 \\
\hline UNIV TORONTO & 70 & 1,1 \\
\hline YALE UNIV & 70 & 1,1 \\
\hline UNIV CALIF BERKELEY & 68 & 1,0 \\
\hline UNIV CALIF SAN FRANCISCO & 64 & 1,0 \\
\hline UNIV MARYLAND & 64 & 1,0 \\
\hline UNIV PITTSBURGH & 64 & 1,0 \\
\hline UNIV WISCONSIN & 64 & 1,0 \\
\hline UNIV CHICAGO & 62 & 0,9 \\
\hline UNIV MI NNESOTA & 61 & 0,9 \\
\hline UNIV PENN & 61 & 0,9 \\
\hline CARNEGIE MELLON UNIV & 60 & 0,9 \\
\hline STANFORD UNIV & 58 & 0,9 \\
\hline KAROLI NSKA INST & 57 & 0,9 \\
\hline INDI ANA UNIV & 55 & 0,8 \\
\hline UNIV CALIF IRVINE & 54 & 0,8 \\
\hline UNIV SO CALIF & 51 & 0,8 \\
\hline DUKE UNIV & 50 & 0,8 \\
\hline TabIa & &
\end{tabular}

Tabla 6. Centros de investigaciónUniversidades con 50 o más artículos indexados en la Web of Science con el descriptor ("social network*).

Estas tablas no necesitan muchos comentarios: Estados Unidos, Inglaterra y Canadá son los responsables de aproximadamente el $70 \%$ de la producción científica en cuestión hoy día.

El panorama es en todo caso prometedor. Pero veamos a continuación cuál es la situación de la revista Social Networks.

\section{La revista Social Networks}

La revista Social Networks presenta la misma tendencia en el número de citas y publicaciones que hemos visto para el conjunto de la disciplina. En la tabla siguiente se puede ver su comparación ventajosa con otras revistas de prestigio como American Anthropologist o American J ournal of Sociology, por ejemplo: 
REDES- Revista hispana para el análisis de redes sociales

Vol.17,\#11, Diciembre 2009

http: // revista-redes.rediris.es

\begin{tabular}{|c|c|c|c|}
\hline & $\begin{array}{c}\text { Social } \\
\text { Networks }\end{array}$ & $\begin{array}{c}\text { American } \\
\text { Anthropologist }\end{array}$ & $\begin{array}{c}\text { American J ournal } \\
\text { of Sociology }\end{array}$ \\
\hline Results found: & 6,564 & 1,903 & 2,092 \\
\hline $\begin{array}{c}\text { Sum of the Times } \\
\text { Cited: }\end{array}$ & 76,419 & 30,673 & 93,939 \\
\hline $\begin{array}{c}\text { Average Citations } \\
\text { per I tem: }\end{array}$ & 11.64 & 16.12 & 44.90 \\
\hline$h$-index: & 101 & 71 & 130 \\
\hline
\end{tabular}

Tabla 7. Artículos, citas y h-index

El h-index, el cual indica el número $\mathrm{h}$ de artículos que tienen al menos $\mathrm{h}$ citas, es muy bueno para Social Networks y, lo que es más importante, crece muy rápidamente. Por lo que se refiere a los autores (tabla no normalizada, así “Borgatti, SP" es diferente de "Borgatti, S"), en la siguiente tabla tenemos el ranking correspondiente:

$\begin{array}{llll}\text { Author } & \text { N } & \text { Author } & \text { N } \\ \text { BORGATII, SP } & 23 & \text { WASSERMAN, S } & 6 \\ \text { EVERETT, MG } & 22 & \text { WELLMAN, B } & 6 \\ \text { BURT, RS } & 21 & \text { WHITE, DR } & 6 \\ \text { DOREI AN, P } & 20 & \text { FRANK, O } & 5 \\ \text { BERNARD, HR } & 14 & \text { KLOVDAHL, AS } & 5 \\ \text { BONACICH, P } & 14 & \text { ROBERTS, JM } & 5 \\ \text { KILLWORTH, PD } & 13 & \text { ROMNEY, AK } & 5 \\ \text { FREEMAN, LC } & 12 & \text { VALENTE, TW } & 5 \\ \text { JOHNSEN, EC } & 12 & \text { BERKOWITZ, SD } & 4 \\ \text { MARSDEN, PV } & 11 & \text { BORGATTI, S } & 4 \\ \text { FAUST, K } & 10 & \text { BREIGER, RL } & 4 \\ \text { FRIEDKIN, NE } & 10 & \text { DUQUENNE, V } & 4 \\ \text { SKVORETZ, J } & 10 & \text { FELD, SL } & 4 \\ \text { MCCARTY, C } & 9 & \text { HAMMER, M } & 4 \\ \text { BOYD, JP } & 8 & \text { HARARY, F } & 4 \\ \text { FARARO, TJ } & 8 & \text { IACOBUCCI, D } & 4 \\ \text { FERLIGOJ, A } & 8 & \text { MIZRUCHI, MS } & 4 \\ \text { KRACKHARDT, D } & 8 & \text { MOODY, J } & 4 \\ \text { SHELLEY, GA } & 8 & \text { PATTISON, P } & 4 \\ \text { BATAGELJ, V } & 7 & \text { PATTISON, PE } & 4 \\ \text { BREWER, DD } & 7 & \text { REITZ, KP } & 4 \\ \text { JOHNSON, JC } & 7 & \text { SEIDMAN, SB } & 4 \\ \text { HUMMON, NP } & 6 & \text { YAMAGUCHI, K } & 4 \\ \text { ROBINS, G } & 6 & \text { BIENENSTOCK, EJ } & 3 \\ \text { SNIJDERS, TAB } & 6 & \text { BUTTS, CT } & 3\end{array}$

Tabla 8. Ranking de los autores de Social networks $(n>2)$

En la Tabla 9 se dispone de las 50 universidades con más artículos publicados en Social Networks y en la tabla 10 el ranking de países. 
REDES- Revista hispana para el análisis de redes sociales Vol.17,\#11, Diciembre 2009

http: //revista-redes.rediris.es

\begin{tabular}{|c|c|}
\hline Institution Name & $\mathbf{N}$ \\
\hline UNIV CALIF IRVINE & 55 \\
\hline UNIV PITTSBURGH & 34 \\
\hline UNIV S CAROLINA & 28 \\
\hline UNIV CALIF SANTA BARBARA & 23 \\
\hline COLUMBIA UNIV & 18 \\
\hline UNIV CALIF LOS ANGELES & 17 \\
\hline UNIV CHICAGO & 16 \\
\hline THAMES POLYTECH & 15 \\
\hline UNIV FLORIDA & 15 \\
\hline UNIV ILLINOIS & 15 \\
\hline UNIV MICHIGAN & 15 \\
\hline HARVARD UNIV & 14 \\
\hline UNIV GRONINGEN & 14 \\
\hline UNIV N CAROLINA & 14 \\
\hline UNIV TORONTO & 13 \\
\hline CARNEGIE MELLON UNIV & 12 \\
\hline UNI V LJ UBLJ ANA & 12 \\
\hline NORTHWESTERN UNIV & 11 \\
\hline UNIV WASHINGTON & 11 \\
\hline BOSTON COLL & 10 \\
\hline UNIV MELBOURNE & 10 \\
\hline AUSTRALI AN NATL UNIV & 9 \\
\hline LOUISIANA STATE UNIV & 9 \\
\hline UNIV CALIF BERKELEY & 9 \\
\hline UNIV AMSTERDAM & 8 \\
\hline ARIZONA STATE UNIV & 7 \\
\hline CORNELL UNIV & 7 \\
\hline E CAROLINA UNIV & 7 \\
\hline UNIV GREENWICH & 7 \\
\hline UNIV MINNESOTA & 7 \\
\hline UNIV SO CALIF & 7 \\
\hline RUTGERS STATE UNIV & 6 \\
\hline STANFORD UNIV & 6 \\
\hline UNIV NEW MEXICO & 6 \\
\hline UNIV UTRECHT & 6 \\
\hline CNRS & 5 \\
\hline SUNY ALBANY & 5 \\
\hline SUNY BINGHAMTON & 5 \\
\hline TILBURG UNIV & 5 \\
\hline UNIV CAMBRIDGE & 5 \\
\hline UNIV STOCKHOLM & 5 \\
\hline BRANDEIS UNIV & 4 \\
\hline CHAPMAN COLL & 4 \\
\hline GEORGE MASON UNIV & 4 \\
\hline GEORGIA STATE UNIV & 4 \\
\hline HONG KONG BAPTIST UNIV & 4 \\
\hline MICHIGAN STATE UNIV & 4 \\
\hline NEW YORK STATE PSYCHIAT INST & 4 \\
\hline PENN STATE UNIV & 4 \\
\hline PORTLAND STATE UNI & \\
\hline
\end{tabular}

Tabla 9. Ranking de Universidades presentes en Social Networks 
REDES- Revista hispana para el análisis de redes sociales

Vol.17,\#11, Diciembre 2009

http: // revista-redes.rediris.es

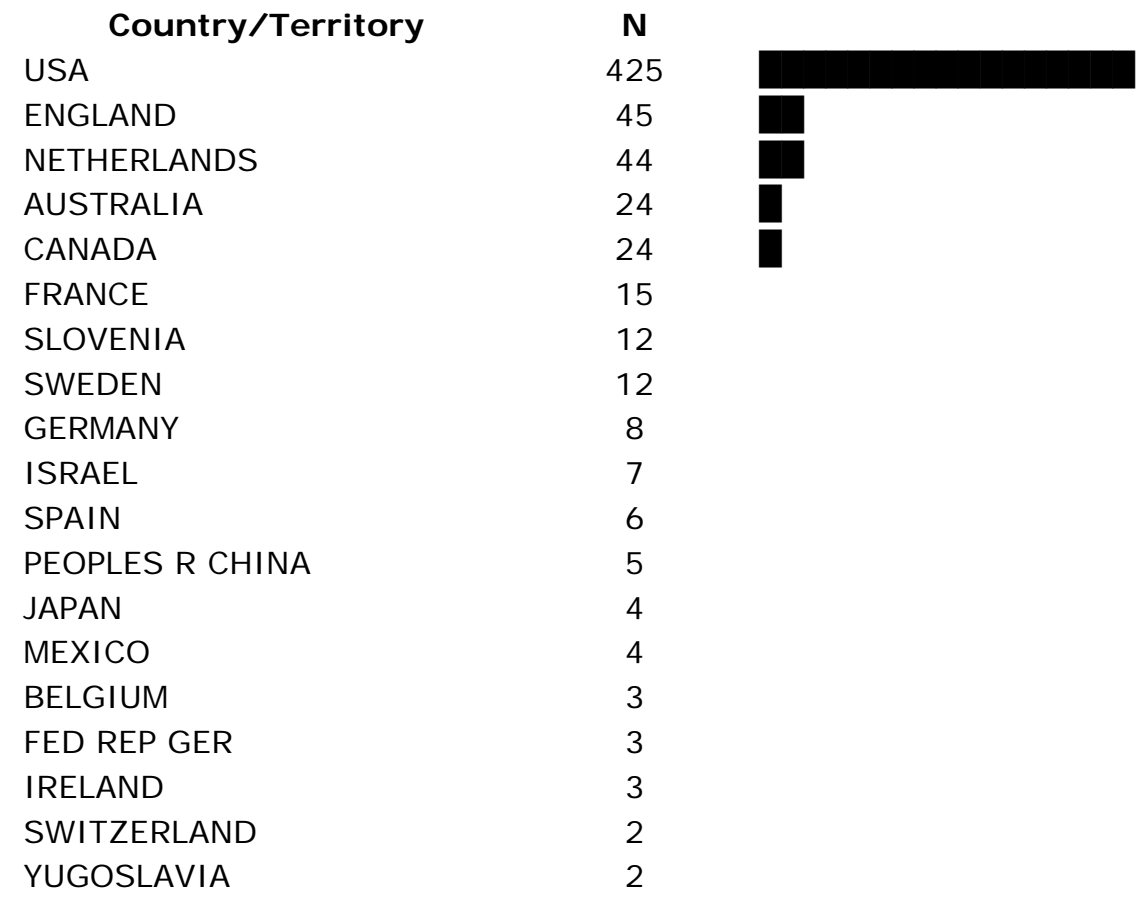

Tabla 10. Ranking de países presentes en Social Networks

La situación es similar a la que ya vimos para el campo de investigación en general, con la excepción de la incorporación de Holanda y Australia (y sus correspondientes instituciones e investigadores) entre los más contribuidores más productivos.

Si analizamos la estructura de colaboración a partir de las co-publicaciones en Social Networks ${ }^{3}$, descubrimos dos grupos de investigadores. Uno, muy amplio y formado por un solo componente. Se trata de investigadores que trabajan temas de metodología relacionada con las redes sociales y ciencias sociales en general... El otro (que se presenta en el centro) constituido por grupos de investigadores que no tienen relación con el resto y que trabajan aspectos desarrollados principalmente con la salud. Por supuesto, no hemos representado los autores que publican solos en este caso ni aquéllos que tienen una sola colaboración. El tamaño indica el grado nodal (número de artículos), la repetición de las colaboraciones viene dada por el grosor de la línea y los colores indican ámbitos muy generales de colaboración.

\footnotetext{
3 Realizada con Ucinet-Netdraw (www.analytictech.com) a partir de los ficheros producidos por los programas gratuitos desarrollados por Loet Leydesdorff para la Web of Science (www.leydesdorff.net).
} 


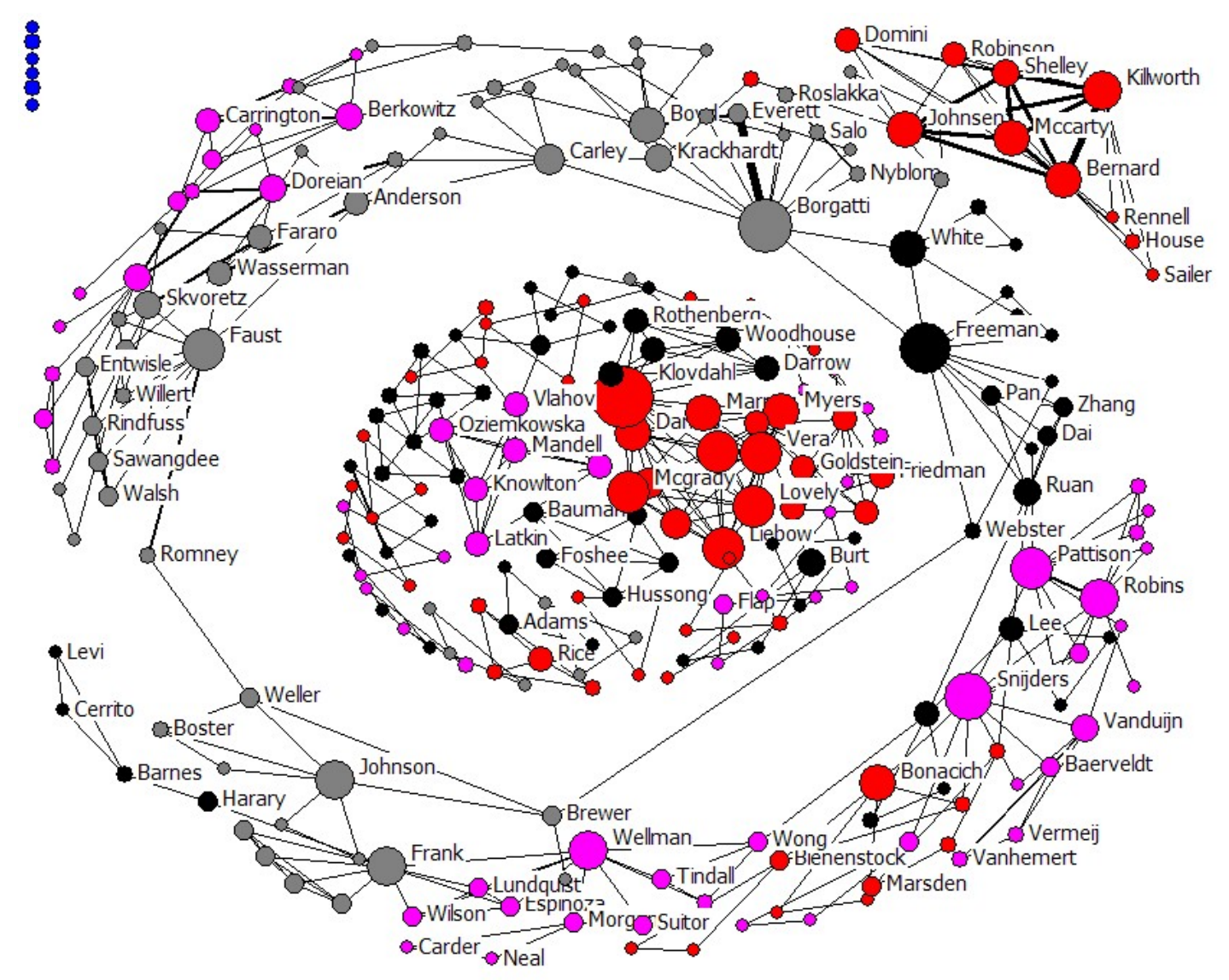

I lustración 4. Estructura de colaboraciones en Social Networks a partir de las copublicaciones presentes en 585 artículos, sin nodos aislados ni "pendants". El tamaño indica el grado nodal y el grosor de la línea la repetición en la colaboración. Los colores distinguen amplios círculos de colaboración.

Esta red da mucho de sí. No tenemos espacio para entrar en detalles, pues ahora nos toca analizar la producción mucho más modesta, pero no por ello menos interesante y prometedora de la Revista REDES.

\section{La revista REDES}

Con la revista REDES hemos hecho la misma operación que con el conjunto de la disciplina: un análisis de contenido con TexStat de sustantivos presentes en los artículos y reseñas publicados hasta la fecha. El resultado se muestra en la tabla 11. 


\begin{tabular}{|c|c|c|c|}
\hline Word & $\mathbf{N}$ & Word & $\mathbf{N}$ \\
\hline social- & 4707 & individuos & 358 \\
\hline red & 3099 & lazos & 347 \\
\hline relacion- & 2965 & estrategi- & 344 \\
\hline organiza- & 1700 & estado- & 333 \\
\hline estructura- & 1138 & cultura- & 332 \\
\hline actor- & 1130 & sociais & 331 \\
\hline grupo- & 1102 & sociologi- & 327 \\
\hline comunidad- & 1031 & amistad- & 318 \\
\hline trabaj- & 875 & tipos & 316 \\
\hline capital- & 863 & agente- & 314 \\
\hline centr- & 846 & cuenta & 312 \\
\hline europ- & 826 & cien- & 308 \\
\hline política- & 796 & ciudad- & 307 \\
\hline local- & 761 & sentido & 303 \\
\hline network & 761 & vida & 303 \\
\hline model- & 724 & acción & 297 \\
\hline forma & 709 & cuando & 297 \\
\hline econom- & 634 & medida & 291 \\
\hline sistema- & 594 & profesion- & 289 \\
\hline vínculo- & 588 & extranjer- & 288 \\
\hline campo- & 585 & http & 286 \\
\hline personal- & 567 & civil & 285 \\
\hline información & 566 & base & 284 \\
\hline recursos & 556 & Press & 279 \\
\hline identi- & 536 & $R$ & 279 \\
\hline institucion- & 518 & conocimiento- & 278 \\
\hline amigo- & 506 & manera & 277 \\
\hline empresa- & 505 & metodologi- & 277 \\
\hline familia- & 473 & confianza & 274 \\
\hline lugar & 362 & micro- & 273 \\
\hline
\end{tabular}

Tabla 11. Análisis de contenido de los ítems publicados en la Revista Redes

De las 10 primeras palabras hemos resaltado en azul las que coinciden con el análisis de contenido realizado para el conjunto de la disciplina y en rojo las que son nuevas. Creo que el resultado también es indicativo. Al lado de los términos propios de la perspectiva de redes (relación, red, estructura, etc.) sigue apareciendo la dimensión comunitaria, pero más desde la intervención social mediante redes que desde la investigación clínica. También, la preocupación por las organizaciones (redes intra y extraorganizacionales) está muy presente.

La Revista REDES forma parte de una red de colaboración más amplia que se manifiesta en el listserv REDES. Para recoger esta estructura de colaboración nada mejor que recurrir a un artículo publicado por Francisco Fernando de la Rosa ( jotro 
REDES- Revista hispana para el análisis de redes sociales

Vol.17,\#11, Diciembre 2009

http: // revista-redes.rediris.es

informático granadino!) en la propia revista en la que se analiza este fenómeno a partir de los datos disponibles en Internet y que ya habíamos analizado unos años antes a partir de las redes personales de algunos miembros de la lista REDES (Ilustraciones 5 y 6 respectivamente).

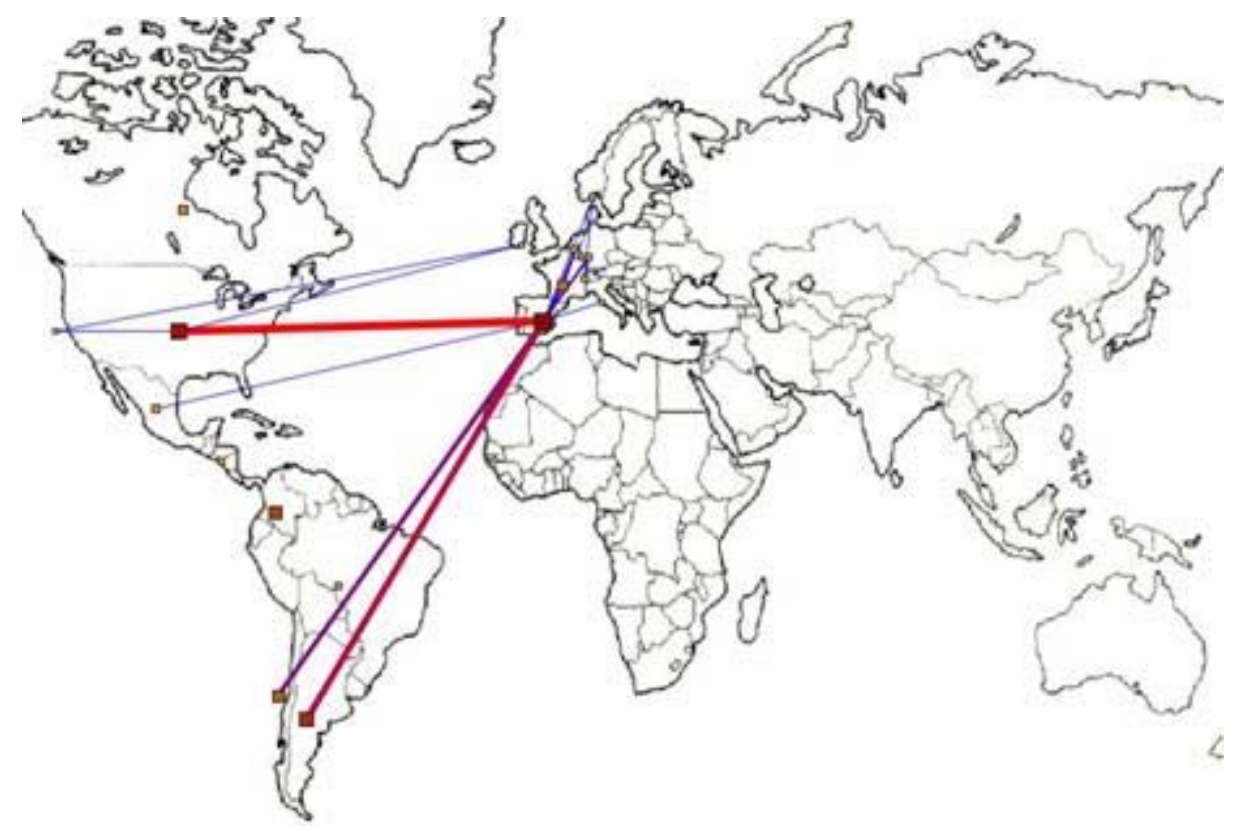

I lustración 5. Localización geográfica de algunos miembros del espacio REDES (de la Rosa et. al, 2007)

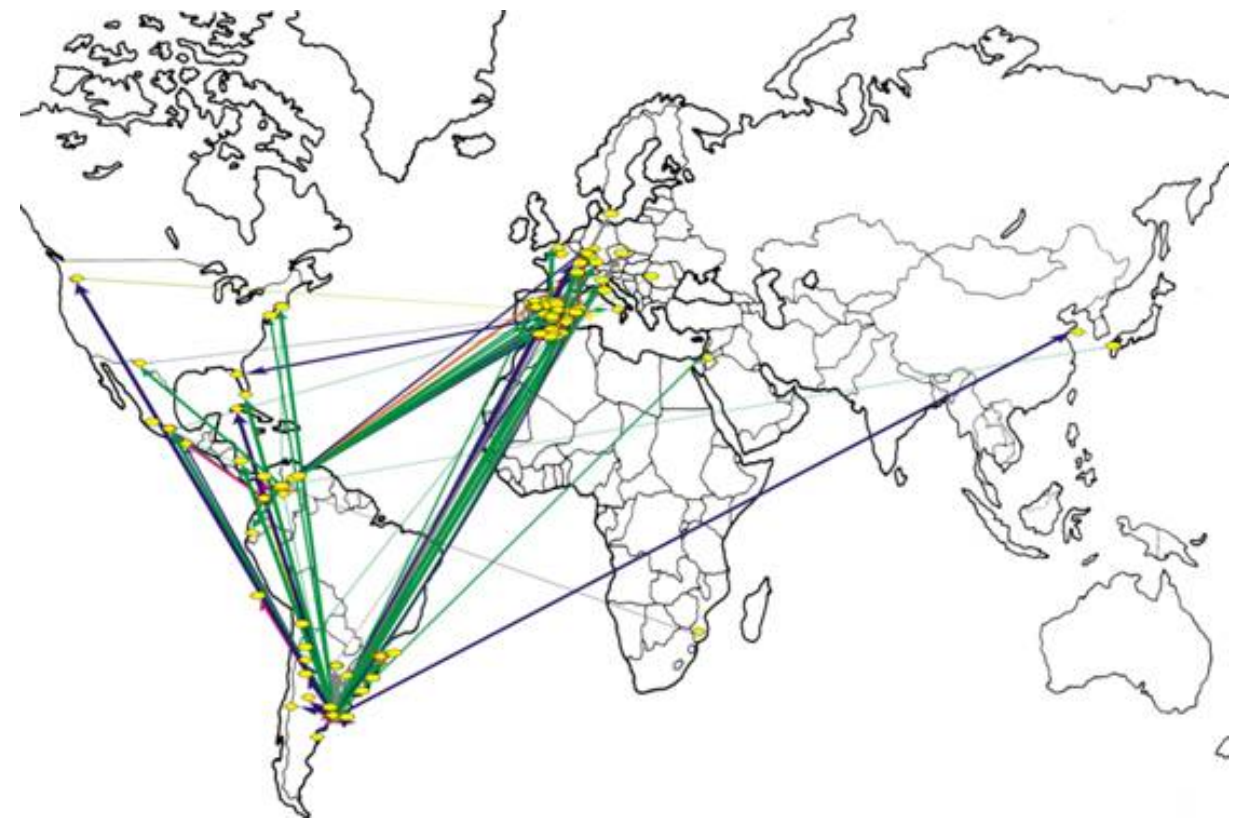

I lustración 6. Localización de las redes personales de algunos miembros del espacio REDES (Molina et. al., 2005).

La existencia de un espacio iberoamericano, potenciado por la reciente incorporación de títulos en portugués, es innegable. 
REDES- Revista hispana para el análisis de redes sociales

Vol.17,\#11, Diciembre 2009

http: // revista-redes.rediris.es

\section{La naturaleza dual de las redes sociales}

Hasta aquí un panorama de la situación existente en la actualidad. Ahora solamente una reflexión. ¿Por qué? ¿Qué tienen las redes que hacen coincidir a campos tan dispares? Creo que la respuesta puede ser en parte la siguiente: las Humanidades (filosofía, historia, literatura...) se caracterizan por abordar sus problemas de investigación con una reducción débil de la realidad y consecuentemente con una operacionalización también débil. Esto no es positivo ni negativo, simplemente es una propuesta de conceptualización de su enfoque. Con las Humanidades no desaparecen las vidas de sus protagonistas, sus afanes, sino que sus fotografías, obras y testimonios están presentes en sus productos.

Por otra parte, las ciencias se caracterizan por realizar reducciones fuertes de la realidad (los modelos) y operacionalizaciones también fuertes para poder medir y poner a prueba sus hipótesis. Pues bien, creemos que las redes se caracterizan por presentar simultáneamente la característica de realizar una reducción débil del fenómeno a estudiar (las redes se nos presentan como naturales, intuitivamente adecuadas) y, al mismo tiempo, una operacionalización fuerte de sus conceptos, debido a las propiedades matemáticas derivadas de la teoría de grafos. Esta doble naturaleza las hace aptas para mediar entre dos mundos a menudo ignorantes el uno del otro.

En el caso concreto que yo conozco, las redes personales, esta baja distancia entre lo percibido por los protagonistas del mundo social y lo obtenido mediante complejas metodologías, esta reducción débil, puede observarse en la llustración 7. El dibujo de Marta de red social sobre su propia red no difiere mucho de la red personal obtenida con Egonet (McCarty et. al. 2007).

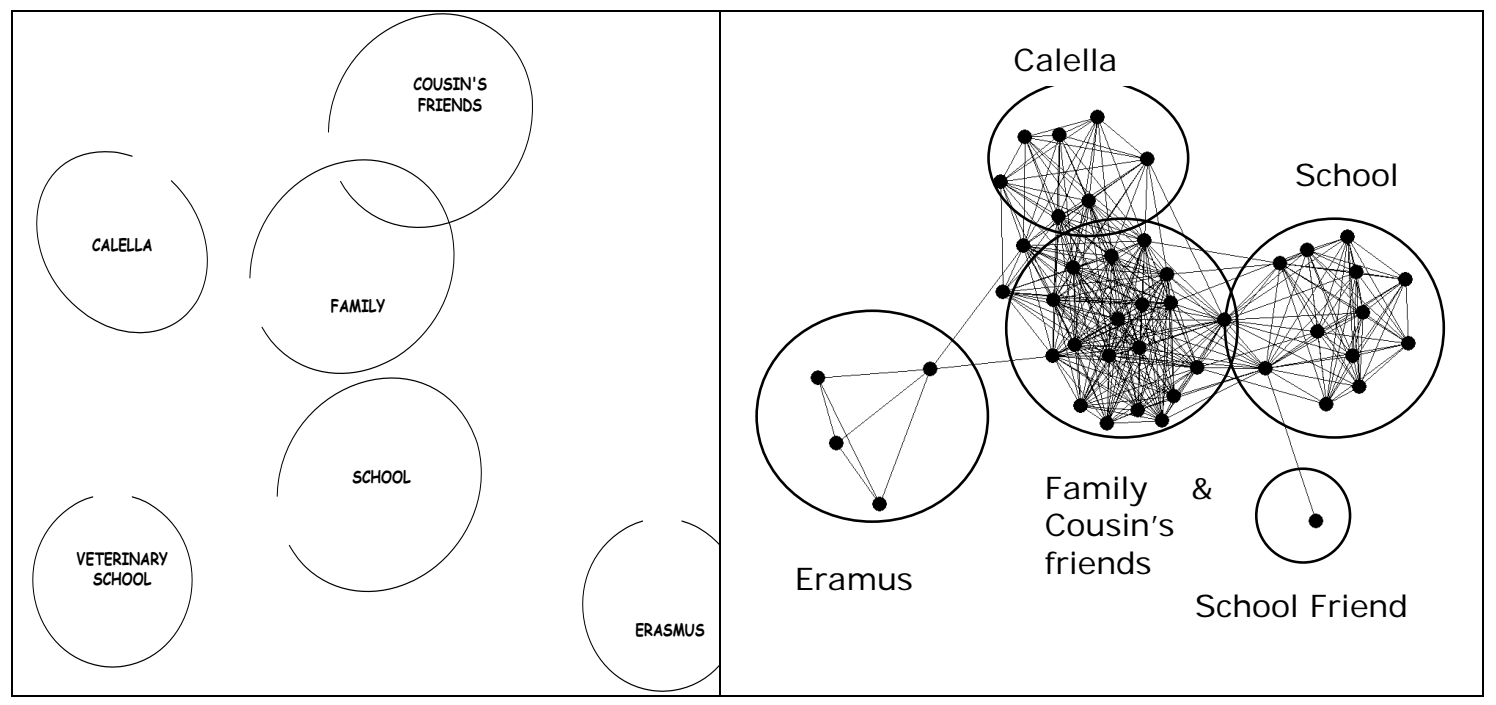

I lustración 7. Comparación del dibujo de Marta sobre su red social y el resultado de Egonet. 
REDES- Revista hispana para el análisis de redes sociales

Vol.17,\#11, Diciembre 2009

http: // revista-redes.rediris.es

Sin embargo, la red social de Marta puede ser descrita completamente con un conjunto preciso de indicadores, en términos de densidad, componentes, centralidades ...

Otro de los efectos de esta reducción débil de las redes es que nos permite rastrear las huellas de la historia, al tiempo que podemos identificar los efectos de las biografías particulares. No por casualidad el nivel representado por las redes personales puede ser conceptualizado como meso, entre lo macro y lo micro (Ferrand, 2002).

Detengámonos un momento en las huellas de la historia. Si observamos las figuras realizada por Juergen Lerner (jotro informático! pero esta vez de la Universidad de Konstanz, Alemania) sobre el conjunto de las redes personales de cubanos en Miami y guineanos en Barcelona ${ }^{4}$, entre otras, observaremos cómo las huellas de la Guerra Fría pueden apreciarse en las redes de los cubanos y cómo historia colonial española puede verse en las redes personales de los guineanos. Tengo que advertir que se trata solamente de un ejemplo ilustrativo, no de un artículo sobre el tema.
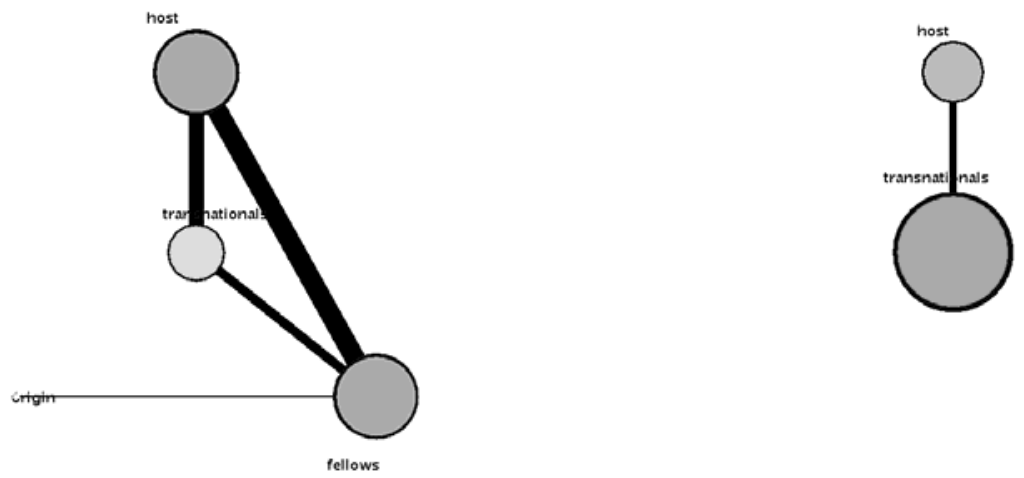

Cubanos en Miami

\section{Guineanos en Barcelona}

I lustración 8. Redes personales agregadas de cubanos y guineanos entrevistados en el proyecto BCS0417429 (www.egoredes.net)

\footnotetext{
${ }^{4}$ Development of a Social Network Measure of Acculturation and its Application to Immigrant Populations in South Florida and Northeastern Spain (BCS-0417429) financiado por la National Science Foundation (con Chris McCarty).
} 
REDES- Revista hispana para el análisis de redes sociales

Vol.17,\#11, Diciembre 2009

http://revista-redes.rediris.es

Cada red personal está representada por cuatro círculos en forma de triángulo relacionados entre sí. En el vértice inferior izquierdo se sitúa el conjunto de personas de la red personal que residen en el país de origen. En el vértice inferior derecho las personas que residen en el país de "destino" pero que son originarios del mismo país que ego. En el centro, la comunidad transnacional encontrada en destino o a partir de la experiencia en destino (ahora sabemos que los nuevos contactos aparecen simultáneamente en origen y en destino, un descubrimiento de Javier Ávila en su investigación doctoral, ver bibliografía). En el vértice superior las personas "nativas" del país de "destino". El tamaño de cada círculo representa la proporción de personas en cada caso. Cuanto más grande sea el círculo, más personas habrá. El color representa la densidad de relaciones dentro de cada círculo. Así un círculo de norteamericanos o españoles (en este ejemplo) muy grande pero blanco significaría que se conocen a muchos "nativos" pero que éstos no tienen relación entre sí. Y esta cuestión es particularmente importante a todos los efectos, como tendremos ocasión de discutir en otra ocasión. Por último el grosor de las líneas indica las relaciones entre los círculos.

Pues bien, en el caso de los cubanos entrevistados las relaciones privilegiadas con los "nativos" norteamericanos, las escasas relaciones con los "transnacionales" y las densas relaciones con los cubanos en Miami son difíciles de explicar sin un apoyo decidido de la Administración estadounidense a este colectivo en relación a otros, como un lance más del gran teatro de operaciones que representó la Guerra Fría y el caso de Cuba (Lubbers et. al. 2007).

Por lo que se refiere a los guineanos, es interesante observar como éstos no tienen contactos en Guinea Ecuatorial: a finales de los 60, con la independencia de la colonia, los estudiantes guineanos becados en España se vieron obligados a elegir entre ambas nacionalidades (eligieron mayoritariamente la española) y rompieron los vínculos con origen. Insistimos que se trata solamente de un ejemplo de cómo la historia deja sus huellas en las redes personales.

Acaba de llegar a mis manos otro interesantísimo ejemplo, en I rán esta vez (Bastani, 2007) de las huellas de la historia en las redes personales: la reislamización de la sociedad iraniana tiene entre otros efectos el aumento de porcentaje de familiares en las redes personales, al tratarse de relaciones menos fiscalizadas y más confiables. 
REDES- Revista hispana para el análisis de redes sociales

Vol.17,\#11, Diciembre 2009

http: // revista-redes.rediris.es

En el futuro seguiremos estas huellas con más detenimiento. Pero hay que mencionar también el efecto en sentido inverso: las huellas de las biografías personales. Observemos la Ilustración 9. Se trata de las redes personales de dos hermanas de origen senegalés, prácticamente con la misma edad, escolarizadas en Cataluña y cataloparlantes. Nada que ver. En el caso de la mujer que aparece en la parte superior, algo más joven que su hermana, la presencia de la familia (en negro; el tamaño indica la intensidad de la relación) es mucho más importante que en el de su hermana, la cual se esfuerza por su parte en mantener espacios separados de relación con personas de otras procedencias.
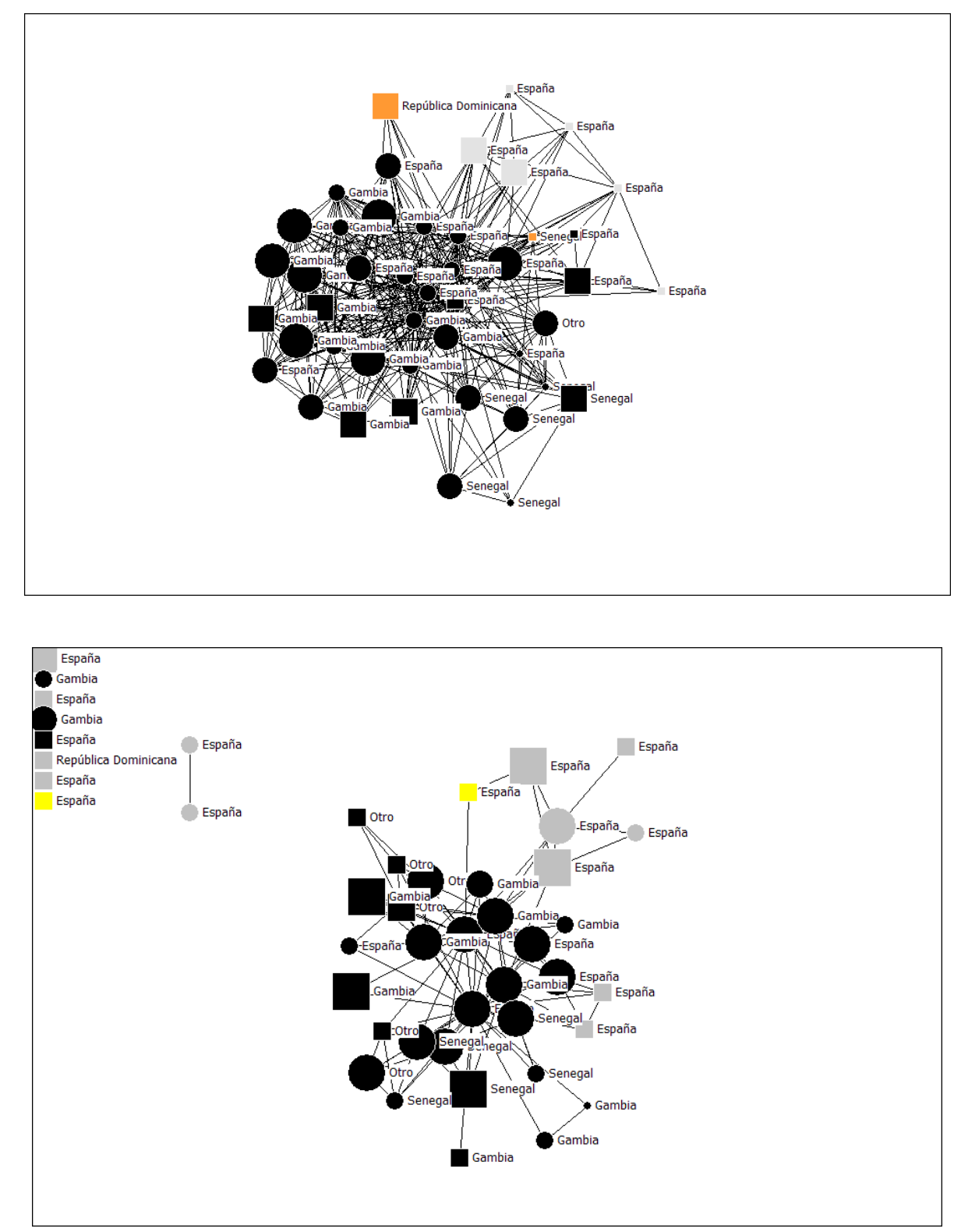

I lustración 9. Redes personales de dos jóvenes mujeres (18 y 20 años respectivamente) escolarizadas en Cataluña y de origen senegalés. 
REDES- Revista hispana para el análisis de redes sociales

Vol.17,\#11, Diciembre 2009

http: // revista-redes.rediris.es

Y nada más. Ahora solamente apuntar que se dan las condiciones para dar un salto adelante en la forma en que concebimos el mundo, para plantearnos las viejas preguntas sobre la sociedad y la cultura desde nuevos puntos de vista. $Y$ ese paso pasa por SIENA y la simulación y análisis dinámicos... pero eso lo vamos a dejar para otra ocasión. Muchas gracias.

\section{Bibliografía}

Ávila Molero, Javier (2007). "Personal networks and ethnic identities. The case of Argentinean immigrants in Catalonia - Spain", XXVII Sunbelt'07 Corfu, Greece May 1-6. < http://seneca.uab.es/antropologia/Egoredes/research/avila.pdf>

Bastani, Susan (2007). "Family comes first: Men's and women's personal networks in Tehran", Social Networks, Volume 29, Issue 3 (357-374).

de la Rosa Troyano, Fco. Fernando \& Rafael Martínez Gasca (2007). "Sistemas de Inteligencia Web basados en redes sociales", REDES. Revista hispana para el análisis de redes sociales, Vol 12, \#9.

Ferrand, Alexis (2002). "Las comunidades locales como estructuras meso", REDESRevista hispana para el análisis de redes sociales, Vol.3,\#4.

Lerner, Juergen \& Ulrik Brandes (2007). "Comparing Networks by their Group Structure with an application to acculturation networks", XXVII Sunbelt'07 Corfu, Greece May 1-6. <http://seneca.uab.es/antropologia/Egoredes/research/juergen.pdf>

Lubbers, Miranda; Molina, J.L. \& Chris McCarty (2007). “Personal Networks and Ethnic Identifications: The Case of Migrants in Spain", International Sociology, en prensa.

McCarty, Christopher; Molina, José Luis; Aguilar, Claudia y Laura Rota (2007). “A Comparison of Social Network Mapping and Personal Network Visualization", Field Methods, Vol. 19 (2) May (145-162).

Milgram, Stanley (1967). "The small world problem", Psychology Today 1 (61-67).

Molina, José Luis (2001). El análisis de redes sociales. Una introducción. Barcelona: Edicions Bellaterra.

Molina, JL; Alejandro A. Ruiz y Laura Teves (2005). "Localizando geográficamente las redes personales", REDES. Revista hispana para el análisis de redes sociales, Vol 8, \#5. 